\title{
Construcción de un índice de arraigo socio- productivo: hacia un concepto amplio de capital social
}

\author{
Hugo Romero Toledo ${ }^{1}$, Omar Barriga ${ }^{2}$ y Guillermo Henríquez ${ }^{3}$
}

\section{Resumen}

El estudio del Capital Social ha tomado fuerza en los últimos años. Sin embargo, el Concepto aun se nos muestra difuso, abarcando una serie de otros conceptos como asociatividad, cooperación, confianza, redes sociales y participación. Para fortalecer al Capital Social proponemos operacionalizar el concepto de arraigo socioproductivo, vinculando el análisis de redes sociales a actividades productivas específicas a través de la construcción de índices.

Palabras clave: Capital social, arraigo, índices.

\section{Abstract}

The study of the Social Capital has taken force in the last years. Nevertheless, the Concept even is to diffuse, including a series of other concepts like associativity, cooperation, confidence, social networks and participation. In order to fortify the Social Capital we propose to operate the concept of socio-productive embeddedness, linking the analysis of social networks to specific productive activities through the construction of index.

Keywords: social capital, embeddedness, indices.

\section{I ntroducción}

El concepto de Capital Social toma fuerza partir de los trabajos Coleman y Putnam desde la década de los 90, con un fuerte énfasis en lo que son las políticas para el Desarrollo. Si bien, no existe una única visión sobre el concepto, se puede establecer algunos consensos en torno a sus dimensiones: asociatividad, cooperación, confianza, redes sociales y participación, que han impulsado, en el caso de Chile, una serie de políticas y programas destinados a identificar y fortalecer las relaciones comunitarias e institucionales en el marco del afianzamiento de la democracia.

\footnotetext{
${ }^{1}$ Sociólogo. Magíster en Investigación Social y Desarrollo, Profesor Colaborador del Departamento de Sociología y Antropología, Universidad de Concepción, Chile.

2 Master en Sociología, PhD en Sociología, Docente del Departamento de Sociología y Antropología, Universidad de Concepción, Chile.

3 Sociólogo, Magíster en Ciencias Sociales, Docente del Departamento de Sociología y Antropología, Universidad de Concepción, Chile.
} 
En el siguiente artículo se plantea una visión teórica y metodológica que pretende complementar el estudio del Capital Social, centrándose en una actividad productiva específica, entendida ésta como el escenario donde se desenvuelven y se relacionan individuos, grupos e instituciones.

\section{Discusión Conceptual}

\section{El Capital Social}

Es necesario clarificar qué es lo que se entiende por Capital Social, ya que muchas veces el concepto es utilizado a nivel político y académico, dando por sentado sus dimensiones y sobreestimando su efecto sobre el comportamiento de personas y grupos. El Capital Social comúnmente aparece dotado de una connotación positiva, casi mística, y asociado a conceptos como redes sociales y participación ciudadana.

Según Durston (2000), el Capital Social constituye un paradigma emergente rico en conceptos que corresponde a realidades sociales altamente relevantes para diseñar programas orientados a promover la participación social y superar la pobreza, en un contexto de insuficiencia del Estado y el Mercado por solucionar los problemas sociales.

En este contexto, se habla de Capital Social en el sentido de un recurso (o vía de acceso a recursos) que en combinación con otros factores, permite lograr beneficios para quienes lo poseen. Esta forma específica de capital residiría en las relaciones sociales (Adler y Kwon, 2002).

Por su parte, Bourdieu (1985) define al Capital Social como el agregado de los recursos reales o potenciales ligados a la posesión de una red durable de relaciones más o menos institucionalizadas de reconocimiento mutuo, mientras que Coleman (1988) lo define como los recursos socio-estructurales que constituyen un activo de capital para el individuo y que facilitan ciertas acciones comunes de los agentes dentro de la estructura (Durston, 2000).

Según Serrano, Alarcón y Tassara (2006) el concepto de Capital Social está ligado a dos grandes dimensiones: al enfoque de vínculos ligado a redes sociales (Granovetter, Atria, Woolcock, entre otros) y el individual y comunitario (Durston), los cuales producen beneficios que pueden organizarse en tres tipos: 1) económicos y materiales, los que permiten acceso a mejores niveles de bienestar; 2) sociales y culturales, los que generan beneficios en el ámbito de la integración social y 3) políticos y cívicos, los que colaboran a alcanzar mayores de cuotas de poder e influencia social. Estos activos son de beneficio directo para los participantes, para la comunidad y para la sociedad en su conjunto (Serrano, et. al, 2006; 13). 
Según el PNUD, se habla de Capital Social para destacar el poder creativo de determinadas formas de organización para dinamizar y potencias la vida social. El PNUD, siguiendo a Putnam, señala que el Capital Social abarca "aquellos rasgos de la organización como confianza, normas y redes que pueden mejorar la eficiencia de la sociedad, facilitando acciones coordinadas" (Putnam, 1993; 167 en PNUD, 2000). Mediante normas de eficiencia y reciprocidad se generan redes de compromiso cívico que contribuyen, en su conjunto, al bienestar.

Para el PNUD, el Capital Social permite: a) compartir la información y disminuir la incertidumbre acerca de la conducta de los otros, b) reducir la tentación de actitudes oportunistas mediante la coordinación, c) gracias al carácter reiterativo de la cooperación, incentivar la prosecución de experiencias exitosas; y d) fomentar una toma de decisión colectiva y así lograr resultados equitativos para todos los participantes (PNUD, 2000; 109).

Más allá de una visión positiva también se hace mención a cómo el Capital Social está presente en asociaciones delictivas, redes de corrupción (Parker, 2001, Granovetter, et. Al, 2005), redes de protección, entre otros.

Por último, se establecen dos dimensiones para el Capital Social, 1) el capital social requiere una red objetiva de vínculos entre individuos, y 2) requiere que los vínculos entre los individuos sean basados en la confianza, reciprocidad y sean emocionalmente positivos (Paxton, 2002).

\section{El Embeddedness (Arraigo)}

El estudio del embeddedness, también traducido como anclaje, incrustamiento o arraigo productivo (Coq Huelva, 2004; Toledo y Romero, 2007), ha tomado fuerza en la investigación social, especialmente norteamericana, desde los años 70, desarrollando y aplicando este concepto a una serie de fenómenos sociales. Sin embargo, los elementos que componen el concepto de arraigo tienen larga data en sociología: la cohesión social, la solidaridad, el comportamiento económico, la toma de decisiones racionales, entre los más importantes.

Originalmente introducido por Polanyi (1947), el concepto de arraigo ha sido fuertemente desarrollado por Granovetter (2005, 2003, 2000, 1995, 1990, 1985) y Uzzi (2005, 2004, 2002, 2000, 1996, 1991), centrándose, principalmente, en como la relaciones sociales influyen sobre la actividad económica, y como la redes sociales pueden sustentar una mejora en los resultados de las acciones de los individuos a través de los vínculos que entre ellos se establecen. 
A partir de estos trabajos se desarrolla la denominada Nueva Sociología Económica, NSE (o NES en inglés) la cuál sostiene, a grandes rasgos, que la economía se construye socialmente, refiriéndose al proceso por el cual las instituciones económicas son producidas, como ellas están amarradas en patrones de comportamiento habituado o sedimentado, y como ellas llegan a ser normalizadas (Peck, 2005).

La perspectiva del arraigo dice relación con la importancia que tienen las redes sociales para la acción. Es decir, reconoce que los actores que están integrados en clusters densos o relaciones múltiples de redes sociales disponen de diferentes conjuntos de recursos y restricciones que aquellos que no están arraigados en tales redes. (Moody y White, 2003). De acuerdo a Granovetter (1985), el comportamiento humano está arraigado en relaciones sociales, capaces de influenciar las decisiones personales. En este sentido, el arraigo se entiende como el contexto por el cual las relaciones sociales se estructuran en redes basadas en la confianza entre los actores, lo que disminuye el riesgo de fraude o delito entre ellos y aumenta considerablemente los temores, sospechas e incertidumbres frente a la llegada masiva de "afuerinos".

Las redes representan proto-instituciones, en el sentido que muchas de ellas, llegarán a ser, subsecuentemente, más estables y regulares con el paso del tiempo. De esta manera es como esencialmente llegan a ser instituciones hacedoras de normas. En la concepción granovetteriana, las redes que fluyen constantemente llegan a ser necesariamente las transportadoras indirectas de relaciones sociales: “Dichas redes son el punto focal para el análisis social, no las grandes estructuras que se encuentran detrás de ellas" (Granovetter, 1985).

En cuanto a la relación de arraigo de las redes sociales locales con el desarrollo social, Uzzi (2000) resalta la idea de que "las redes de organización operan sobre una lógica de intercambio que difiere de la lógica de los mercados." Se dice que esta lógica de intercambio está arraigada, cuando los vínculos sociales que se realizan incluyen las expectativas y oportunidades de los actores, de manera que difiere de la lógica del comportamiento económico del mercado.

Un aspecto muy importante de considerar en el reconocimiento del arraigo es que las inversiones en las redes se centra en los lazos establecidos entre las organizaciones y sus miembros, basadas en que se conocen unos con otros desde los círculos sociales, tales como: compañeros de trabajo o de escuela, amigos o parientes. Como redes de terceras partes (redes más lejanas que el contacto diádico) los lazos previos permiten recursos y expectativas desde una relación 
existente para ser comprometida en una nueva relación o para elaborar la multiplicidad de las relaciones (Uzzi, 2000).

Esta perspectiva, basada en la teoría de redes sociales, argumenta que el arraigo lleva las motivaciones de los actores más allá del estrecho objetivo de las ganancias económicas inmediatas hacia el enriquecimiento de relaciones a través de la confianza y la reciprocidad. La confianza ayuda a reducir las incertidumbres transaccionales y crea oportunidades para el intercambio de bienes y servicios escapando de lo lógica de precios o contratos.

Siguiendo principalmente a Granovetter (2005) y a Uzzi (2000) el arraigo vincula funciones de desempeño únicas y tiene tres rasgos principales:

- Confianza: Entendido como un mecanismo de gobernabilidad único, ya que promueve intercambios voluntarios (no obligatorios) de bienes y servicios entre los actores, facilitando la extensión de beneficios a los socios de las transacciones e invitando al socio que recibe a una acción reciproca cuando surja una nueva situación. Incluso puede llegar a promover el intercambio de bienes o servicios críticos (escasos e importantes para un grupo o comunidad, por ejemplo, los recursos naturales). Junto con lo anterior, la confianza permite adaptarse frente a problemas no previstos de una forma que es difícil de alcanzar a través de los vínculos de "brazos largos". Según Granovetter (1973), las vinculaciones de brazos cortos son las que relacionan estrechamente a los individuos al interior de las redes familiares, laborales, comunitarias, etc. Los vínculos de brazos largos relacionan al conjunto de redes de las que forman parte los individuos (bajos en intención pero altos en extensión) y son primordiales para resolver situaciones no cotidianas ni regulares, tales como buscar empleo o acceder a las instancias de información y toma de decisiones.

- Transferencia de información refinada: Incluyendo el know how tácito y estratégico que prepara a las empresas en eficacia transaccional y en su capacidad de responder al entorno. Esta transferencia de información está fuertemente asociada a la identidad de los individuos y la calidad de su vínculo social, haciendo a la misma creíble, interpretable y de calidad.

- Solución de problema en conjuntos: Permite a los actores coordinar funciones, proveyendo de retroalimentaciones (feedback) más rápidos y explícitos que los mecanismo basados en el mercado (Hirchman 1970, en Uzzi, 2000). Lo anterior permite a las empresas u organizaciones acelerar el aprendizaje y corrección de los problemas. 
Un elemento relevante a tener en cuenta dice relación con el peso creciente que asumen las relaciones económicas en el comportamiento de los actores sociales: “así como las transacciones económicas están arraigadas en relaciones sociales, las nuevas relaciones sociales están arraigadas en transacciones económicas: las personas en los negocios y las organizaciones en general comprenden que para ellas lo importante es obtener cada vez más y mejores beneficios (Uzzi, 1996).

Según Wellman (1995), los lazos y redes sociales además tienen ciertas características que se complementan con el concepto de arraigo:

- Los lazos son, por lo general, asimétricamente recíprocos, variando en contenido e intensidad.

- Los lazos vinculan a los miembros de una red en forma directa, e indirecta. Por tanto, deben ser definidos en el contexto de estructuras de redes más grandes.

- La estructuración de los lazos sociales crea redes no aleatorias, por tanto, conglomerados, límites y vínculos cruzados.

- Los vínculos que cruzan conectan los conglomerados como también a los individuos.

- Los lazos asimétricos y las redes complejas distribuyen los recursos escasos en forma diferencial.

- Las redes estructuran las actividades colaborativas y competitivas para asegurar los recursos escasos.

\section{Reconceptualización del Arraigo}

Sostenemos que es necesaria la reconceptualización del concepto de embeddedness, aquí traducido como arraigo. Si bien, se pretende respetar su significado, creemos que es conveniente dar un paso más y ligarlo directamente a la producción.

De esta forma, nuestro concepto de arraigo socio-productivo consiste en entender las relaciones sociales en un contexto productivo específico, es decir, que las formas de coordinación y asociatividad están condicionadas por las actividades productivas que las personas o los grupos realizan. Dicho de otra manera, la forma en que las redes sociales (y los recursos que se mueven a través de ella) dependen de la actividad productiva que se desarrollen en territorios determinados. 
Los grupos sociales se identifican con las actividades productivas que realizan. Mineros, pescadores, agricultores, campesinos, obreros industriales, académicos, funcionarios públicos y privados, y empresarios, son miembros de redes complejas que los vinculan mediante sus actividades particulares, con otras personas que indirectamente se articulan a dichos dinamismos, como también con el resto de las actividades que se realizan en determinados territorios (Romero, 2007).

Para entender el Capital Social es necesario ver a que se dedica la gente, en que actividades productivas se basan las redes sociales, el tiempo en que la persona o el grupo social a estado ligado a dicha actividad. Solo de esa forma se puede entender la fuerza y función de los vínculos y entender el potencial de conceptos como el de soporte social.

Según Wellman (1990) del Instituto de Redes Sociales de la Universidad de Toronto, soporte social es cuando los miembros de la comunidad proveen principalmente la forma de obtener recursos de la gente y los vecinos, desde los intercambios comerciales, la distribución institucional y apropiaciones coercitivas. Los amigos y parientes entregan el soporte social más allá de la reciprocidad, y en el diario vivir se accede a diversas oportunidades y se reduce la incertidumbre. En estos espacios sociales, se sostienen intercambios informales para la expansión y reproducción de la supervivencia vecinal. El soporte social es lo que sostiene a la comunidad.

En nuestra teorización, al estudiar las redes ligadas a una actividad productiva específica, entendida ésta como el espacio social laboral de interacción cotidiana, pueden entenderse el tipo de vinculaciones que tendrán los miembros de una red. La posición en el sistema productivo condiciona el tipo de recursos que transitan por la red. No es lo mismo un grupo de ex alumnos de Ingeniería Comercial vinculados a empresas transnacionales, que un grupo de ex alumnos de un liceo vinculados a actividades de extracción de materias primas.

El soporte social represente los pilares en los que descansa una red densa, y que apoyan a sus miembros frente a distintos requerimientos. Este concepto es sumamente importante, y se desarrolla fuertemente con la reproducción simbólica del grupo y del individuo. Dicho en términos sencillos: Debemos apoyarnos todos para que nos vaya bien.

Indudablemente la importancia de estos conceptos radica en el potencial de desarrollo social, humano y personal, toda vez que entendemos que un individuo está arraigado a un círculo cercano de familiares, amigos y compañeros de trabajo que mantienen entre todos un fuerte relación recíproca de beneficios. 


\section{I. Datos y Método}

\section{Medición del Capital Social en Chile}

A continuación se presentan 3 ejemplos de cómo ha sido medido el Capital Social en Chile. A través de distintos subíndices e índices, distintos cientistas sociales han intentado, de forma loable, darle un sustrato empírico-analítico a las dimensiones del concepto de Capital Social.

EI PNUD (2000) construyó un Índice de Capital Social Formal, tomando en cuenta las siguientes dimensiones: confianza social, compromiso cívico y normas de reciprocidad. A partir de una encuesta realizada por dicho organismo el año 1999, se recogió información sobre la confianza en las personas y en las instituciones

gubernamentales y civiles (Iglesia y Sindicatos). El compromiso cívico se midió a través de las siguientes preguntas: ¿Cuan justificable o injustificable es para usted que las personas: - Compren algo que saben que es robado?, - Acepten sobornos en el desempeño de sus deberes? - Tiren basura en lugares públicos?, engañen en el pago de impuesto si pueden? Por último, las normas de reciprocidad fueron medidas a través de las siguientes variables: reconocimiento, percepción de ser tomado en cuenta, y la distribución de los beneficios del crecimiento económico.

La medición del Capital Social Informal se basó en una adecuación de las variables anteriores, rescatando, por ejemplo a la conversación como un indicador de confianza; la reciprocidad fue operacionalizada a través de la percepción del trabajo y la amistad. El compromiso cívico informal se operacionalizó a través de preguntas de denuncia sobre maltrato infantil.

Serrano, Alarcón y Tassara (2006) generaron un Índice de Capital Social, en el marco de una consultoría, para tres barrios urbanos de Coquimbo. Las dimensiones utilizadas para medir el Capital Social fueron las siguientes: Información, Participación, Redes, Confianza, Empoderamiento y Liderazgo, Ciudadanía y Cultura Cívica y Acción Colectiva para la Provisión de Servicios/ Recursos.

Parker (2001), en un estudio sobre el Capital Social en jóvenes secundarios, operacionalizó el concepto a partir de los siguientes indicadores: niveles de confianza mutua, redes sociales (operacionalizada como: ¿a quién acudiría en caso en necesidad?) y participación en organizaciones.

\section{Construcción del Í ndice de Arraigo Socio-Productivo}

Los datos con los que se construyó el índice fueron extraídos de la Encuesta de Caracterización Económica y Social de la VIII Región tomada ente los meses de junio y noviembre de 2002, en el contexto del Proyecto del Fondo Nacional de 
Desarrollo Regional (FNDR) № Código BIP № 20183334 - 0. La muestra es de 1329 Jefes de Hogar, de los cuales 1086 son hombres y 243 son mujeres, todos ligados a la actividad pesquera.

\begin{tabular}{|l|l|r|r|r|r|r|}
\hline \multirow{2}{*}{} & \multicolumn{4}{|c|}{} & \multirow{2}{*}{} \\
\cline { 3 - 7 } & \multicolumn{2}{c|}{$\begin{array}{c}\text { Flota } \\
\text { Artesanal }\end{array}$} & $\begin{array}{c}\text { Flota } \\
\text { Industrial }\end{array}$ & $\begin{array}{c}\text { Planta de } \\
\text { Consumo } \\
\text { Humano }\end{array}$ & $\begin{array}{c}\text { Planta de } \\
\text { Reducción }\end{array}$ & \multirow{2}{*}{ Total } \\
\hline $\begin{array}{l}\text { Segmento muestra } \\
\text { SEXO }\end{array}$ & Hombre & 282 & 354 & 129 & 321 & 1086 \\
\cline { 2 - 7 } & Mujer & 0 & 2 & 218 & 23 & 243 \\
\hline \multicolumn{2}{|l|}{ Total } & 282 & 356 & 347 & 344 & 1329 \\
\hline
\end{tabular}

Tabla 1. Distribución por Sexo y Sector de Empleo en la Actividad Pesquera

De los 1086 hombres, 235 se encontraban cesantes al momento de realizar la encuesta. En el caso de las mujeres, 88 estaban en la misma situación. Por tanto la distribución rescata el sector en el cual las personas se han empleado en términos del oficio que realizan o han realizado. Junto con lo anterior, la población encuestada lleva en promedio 14 años vinculados a la actividad pesquera, y solo un $25 \%$ lleva más de 18 años vinculado a ésta actividad.

El Índice de Arraigo Socio-Productivo que propongo se construye a partir de 3 dimensiones:

Participación. Operacionalizada en los siguientes indicadores:

- Sindicato del encuestado.

- A cual otro sindicato pertenece.

- Primera organización nombrada.

- Segunda organización nombrada.

- Tercera organización nombrada.

La participación a nivel a Sindical casi el $49 \%$ de los entrevistados menciona la participación en al menos un sindicato. Con una test ANOVA y una prueba Tukey, se muestra que desde los 12 años de experiencia en la actividad pesquera se incentiva la participación en sindicatos, lo cual es un buen indicador de que la actividad productiva fomenta el capital social.

Con respecto a la pertenencia a organizaciones, solo 579 personas declaran pertenecer a una organización, es decir, cerca del $44 \%$ de los encuestados, la cifra disminuye hasta llegar a 7 personas, quienes declaran pertenecer a una tercera organización. 
La población, en general, participa por sobre todo de Asociaciones Deportivas (como primero organización 348 personas, como segunda organización 25 personas), seguida de Asociaciones Religiosas, sobre todo Evangélicas (como primer organización 146 personas, como segunda organización 22 personas), y muy atrás en Asociaciones Vecinales (como primer organización 43 personas, como segunda organización 11 personas). Al mismo tiempo, muy poca gente se dedica al voluntariado, y solo 1 persona pertenece a un partido político.

Por la participación en alguna organización se asigna 1, por la no participación 0. Luego los valores fueron promediados por cada caso y multiplicado por 5 (número de indicadores). Mientras más alto el valor, mayor participación. El subíndice de participación va de 0 (nula participación) a 5 (alta participación).

Se realizó un Análisis de Componentes Principales (ACP) con rotación varimax, donde en un factor se cargó la participación en sindicatos y en el otro la participación en organizaciones, sin embargo, se tomó la decisión de darles el mismo peso, ya que ambos tipos de participación están consideradas dentro del concepto de Capital Social.

\begin{tabular}{|l|c|c|}
\hline & \multicolumn{2}{|c|}{ Component } \\
\hline & 1 & 2 \\
\hline Pertenencia a Sindicato &, 136 &, 785 \\
\hline $\begin{array}{l}\text { Pertenencia a un } \\
\text { segundo Sindicato }\end{array}$ &, 388 &, 624 \\
\hline $\begin{array}{l}\text { Pertenencia a 1 } \\
\text { organización }\end{array}$ &, 591 &,- 051 \\
\hline $\begin{array}{l}\text { Pertenencia a 2 } \\
\text { organizaciones }\end{array}$ &, 771 &,- 263 \\
\hline $\begin{array}{l}\text { Pertenencia a 3 } \\
\text { organizaciones }\end{array}$ &, 627 &,- 185 \\
\hline
\end{tabular}

Tabla 2. Matriz de Componentes

Extraction Method: Principal Component Analysis. a 2 components extracted.

\begin{tabular}{|l|c|c|}
\hline & \multicolumn{2}{|c|}{ Component } \\
\hline & \multicolumn{1}{|c|}{1} & \multicolumn{1}{c|}{2} \\
\hline Pertenencia a Sindicato &,- 099 &, 790 \\
\hline $\begin{array}{l}\text { Pertenencia a un } \\
\text { segundo Sindicato }\end{array}$ &, 190 &, 710 \\
\hline $\begin{array}{l}\text { Pertenencia a 1 } \\
\text { organización }\end{array}$ &, 580 &, 124 \\
\hline $\begin{array}{l}\text { Pertenencia a 2 } \\
\text { organizaciones }\end{array}$ &, 814 &,- 027 \\
\hline $\begin{array}{l}\text { Pertenencia a 3 } \\
\text { organizaciones }\end{array}$ &, 654 &, 006 \\
\hline
\end{tabular}

Tabla 3. Matriz de Componentes Rotados

Extraction Method: Principal Component Analysis. Rotation Method: Varimax with Kaiser Normalization.

a Rotation converged in 3 iterations.

Soporte Social. Operacionalizado en los siguientes indicadores.

- Si necesita dejar hijos con adultos

- Si necesita dinero para pan y leche

- Si necesita \$250.000 para emergencia medica

- Si se quedo sin trabajo 
- Si algún miembro del hogar esta siendo agredido

- Si necesita cuidar de un familiar enfermo

- Si necesita contarle a alguien sus penas

- Si en su casa se presentan problemas de alcoholismo y/o drogadicción

Las opciones de respuesta van desde: $0=$ Nadie, $1=$ Sí mismo, $2=$ Familia Nuclear, $3=$ Familia Extendida, $4=$ Vecinos, $5=$ Organizaciones Comunitarias, $6=$ Organizaciones Estatales y $7=$ Otros. Es decir, desde el círculo o redes más cercanas hasta redes más lejanas, de carácter administrativo-gubernamental.

Se realizó un ACP identificándose dos nuevas variables. 1) Relaciones de Brazos Cortos, donde se agrupan indicadores de ayuda social directa; y 2) Relaciones de Brazos Largos, donde estaban los indicadores relacionados con agresión en el hogar y problemas de alcoholismo y/o drogadicción, que deben ser resueltas por organizaciones e instituciones.

Por lo tanto, mientras menor es el valor, más soporte social de los círculos cercanos. Los resultados fueron promediados para cada caso, y fueron ponderados por los indicadores que componen las nuevas variables (6 para las de Brazos Cortos y 2 para las de Brazos Largos)

Arraigo. Operacionalizado por los siguientes indicadores.

- Estatus Laboral

- Inscripción en los registros del Servicio Nacional de Pesca (SERNAPESCA) por parte el encuestado

- Años de experiencia laboral sector pesquero

El indicador Estatus Laboral se dicotomizó en $0=$ Cesante y $1=$ Trabajando; de la misma forma, la inscripción en los registros de SERNAPESCA, se trabajó con $0=$ No inscrito y $1=$ Inscrito. En el caso del indicador años de experiencia laboral en el sector pesquero, se elaboraron 6 rangos con un amplitud de 4 años, siguiendo el criterio indicado por las frecuencias y el promedio.

Estos 3 indicadores fueron sometidos a un ACP, cargando todos en un componente. Por tanto, el Subíndice de Arraigo, Indicando los valores cercanos a 8 el máximo nivel de arraigo con la actividad productiva.

Desarrollo Personal. Operacionalizado como:

- Siente que es una persona tan valiosa como otras con que trabaja

- Se siente exitoso 
- Siente que tiene buenas cualidades

- Se siente contento con si mismo

- Se siente capaz de hacer las cosas tan bien como otras personas

- Tiene una actitud positiva hacia si mismo

Todos estos indicadores van desde $1=$ Siempre, $2=$ Casi Siempre, $3=\mathrm{A}$ veces, $4=$ Casi Nunca, $5=$ Nunca. Se realizó una ACP, quedando los 6 indicadores en un mismo componente.

Por tanto, los valores de los indicadores para cada caso fueron promediados lo que se traduce que aquellos casos con menores valores son los que tienen más alto desarrollo personal.

Entonces, hemos generado 4 subíndices, a saber: Soporte Social, Arraigo, Participación, y Desarrollo Personal.

Estos 4 subíndices fueron sometidos a un ACP, generándose dos nuevas variables:

\begin{tabular}{|l|l|r|}
\hline \multicolumn{2}{|l|}{$\begin{array}{l}\text { Kaiser-Meyer-Olkin Measure of } \\
\text { Sampling Adequacy (KMO). }\end{array}$} &, 510 \\
\hline $\begin{array}{l}\text { Bartlett's Test } \\
\text { of Sphericity }\end{array}$ & $\begin{array}{l}\text { Approx. Chi- } \\
\text { Square }\end{array}$ & 115,98 \\
\cline { 2 - 3 } & df & 0 \\
\cline { 2 - 3 } & Sig. &, 000 \\
\hline
\end{tabular}

Tabla 4. KMO y Test de Bartlett.

El KMO es una medida para cuantificar el grado inter-correlaciones entre las variables y la conveniencia del análisis factorial. En nuestro caso, el KMO es moderado (alto es sobre 0,7 ), pero según la literatura, aun así puede considerarse seguir con análisis.

La prueba de Bartlett se utiliza para verificar si la matriz de correlaciones es una matriz identidad, en cuyo caso no existirían correlaciones entre las variables y el modelo factorial no sería adecuado para explicar causa comunes (Vivo, et al. 2005). En este caso se rechaza dicha hipótesis nula.

\begin{tabular}{|l|r|r|}
\hline & \multicolumn{1}{|c|}{ Inicial } & Extraction \\
\hline Indisoport & 1,000 &, 440 \\
\hline Indearriago & 1,000 &, 652 \\
\hline Indepart & 1,000 &, 631 \\
\hline Indedesper & 1,000 &, 584 \\
\hline
\end{tabular}

Tabla 5. Comunalidades 
La tabla de comunalidades representa en cada una de las variables la proporción de su varianza que queda explicada por los factores comunes del modelo factorial obtenido. En este caso, todas las variables quedan suficientemente explicadas.

\begin{tabular}{|l|r|r|}
\hline \multirow{2}{*}{} & \multicolumn{2}{|c|}{ Component } \\
\cline { 2 - 3 } & 1 & \multicolumn{1}{|c|}{2} \\
\hline indisoport &, 305 &,- 589 \\
\hline indearriago &, 743 &, 317 \\
\hline indepart &, 785 &, 125 \\
\hline indedesper &,- 209 &, 735 \\
\hline
\end{tabular}

Tabla 6. Matriz de Componente.

Extraction Method: Principal Component Analysis.

a. 2 components extracted.

\begin{tabular}{|c|c|c|}
\hline \multirow{2}{*}{} & \multicolumn{2}{|c|}{ Component } \\
\cline { 2 - 3 } & 1 & 2 \\
\hline indisoport &, 098 &,- 656 \\
\hline indearriago &, 805 &, 059 \\
\hline indepart &, 783 &,- 136 \\
\hline indedesper &, 040 &, 763 \\
\hline
\end{tabular}

Tabla 7. Matriz de Componentes Rotados.

Extraction Method: Principal Component Analysis.

Rotation Method: Varimax with Kaiser Normalization.

a Rotation converged in 3 iterations.

\begin{tabular}{|c|c|c|c|}
\hline & Total & $\%$ Varianza & \% Acumulado \\
\hline Componente 1 & 1,273 & 31,83 & 31,813 \\
\hline Componente 2 & 1,035 & 25,864 & 57,677 \\
\hline
\end{tabular}

Tabla 8. Varianza Total Explicada

La matriz de componentes rotados muestra la correlación de cada uno de los índices construidos con los 2 componentes resultantes del proceso de análisis. Como se aprecia, en el primer componente existen dos correlaciones altas y positivas, Arraigo y Participación; mientas que en el segundo componente Soporte Social tiene una correlación alta e inversa, mientras que Desarrollo Personal tiene una correlación alta y positiva.

Por su parte, la Tabla 8, indica el porcentaje de variación retenido por cada componente. 


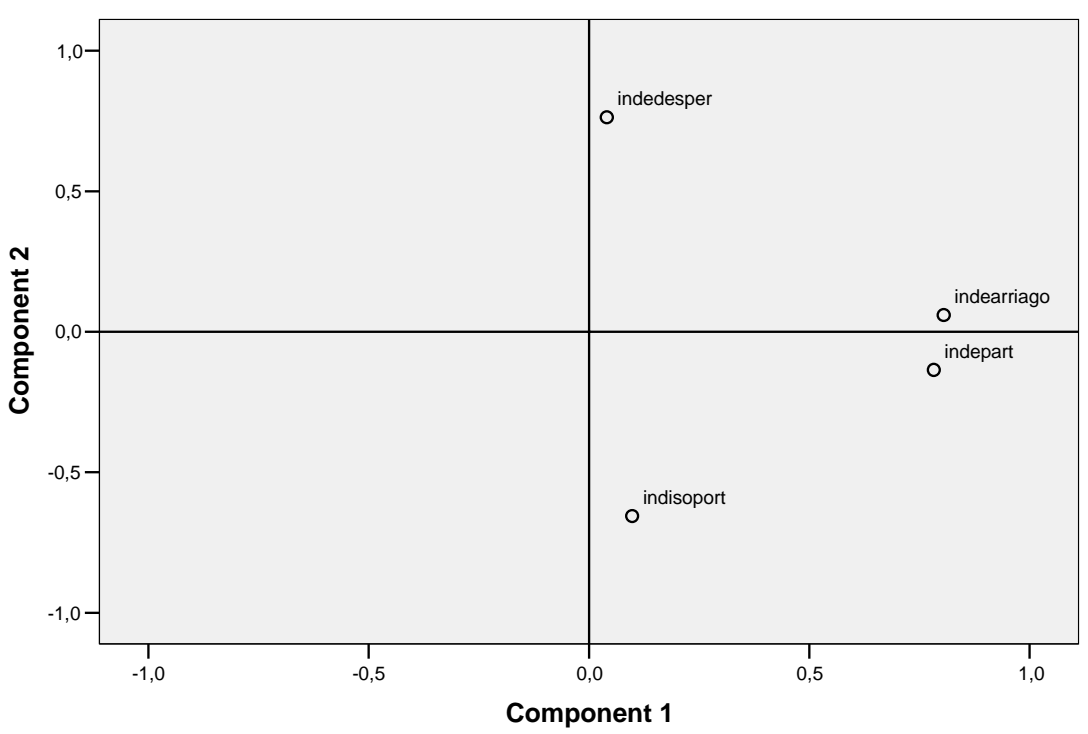

I lustración 1. Componentes Rotados.

Estas dos nuevas variables latentes se traducen en dos Índices: a) Por un lado está la variable Arraigo Participación-Productivo, compuesta por los subíndices Arraigo (con una correlación alta y positiva $=0,805$ ) y Participación (con una correlación alta y positiva $=0,783) ; \mathrm{y}$, b) la variables Arraigo Social, compuesta por los subíndices Desarrollo Personal (con una correlación alta y positiva $=0,763$ ) y Soporte Social (con una correlación un poco más moderada e inversa $=-0,656$ ).

Estas variables fueron transformadas en índices mediante un promedio de los subíndices que las componen. Posteriormente se realizó un último $A C P$, donde ambos índices cargaron en un solo componente. Esta nueva variable latente fue denominada Arraigo Socio-Productivo, y su correspondiente índice fue generado a partir de un promedio entre el índice Arraigo Productivo y Arraigo Social.

El Índice de Arraigo Socio-Productivo queda entonces compuesto por 2 subíndices. En el se plasman 4 grandes elementos de la hora de comprender al Capital Social: 1) la participación ligada a organizaciones de la sociedad civil; 2) los años de experiencia ligados a la actividad y la formalidad de esta participación; 3) el soporte social que ocupa la comunidad frente a determinados problemas; y 4) la percepción-valoración que tienen los individuos sobre sí mismos. 


\begin{tabular}{|l|r|r|r|r|r|r|}
\hline \multirow{2}{*}{ Component } & \multicolumn{3}{|c|}{ Initial Eigenvalues } & \multicolumn{3}{|c|}{ Extraction Sums of Squared Loadings } \\
\cline { 2 - 7 } & \multicolumn{1}{|c|}{ Total } & \% of Variance & Cumulative \% & Total & \% of Variance & Cumulative \% \\
\hline 1 & 1,039 & 51,973 & 51,973 & 1,039 & 51,973 & 51,973 \\
\hline 2 &, 961 & 48,027 & 100,000 & & & \\
\hline
\end{tabular}

Tabla 9. Total de Varianza Explicada.

Extraction Method: Principal Component Analysis.

\begin{tabular}{|c|c|c|}
\hline & Initial & Extraction \\
\hline $\begin{array}{c}\text { Arraigo de Participación- } \\
\text { Producción }\end{array}$ & 1,000 &, 520 \\
\hline Arraigo Social & 1,000 &, 520 \\
\hline
\end{tabular}

Tabla 10. Comunalidades.

\begin{tabular}{|c|c|}
\hline & Component 1 \\
\hline $\begin{array}{c}\text { Arraigo de Participación- } \\
\text { Producción }\end{array}$ &, 721 \\
\hline Arraigo Social &, 721 \\
\hline
\end{tabular}

Tabla 11. Matriz de Componentes.

Por último, el promedio entre Arraigo de Participación-Producción y Arraigo Social fue multiplicado por 2 (número de variables utilizadas), quedando ordenados de 19 a 94, siendo los números más altos correspondiente a los casos de mayor arraigo.

\section{I I. Presentación de Resultados}

Desde un punto de vista descriptivo, el Índice de Arraigo Socio-Productivo tiene una media de 0,50, con una desviación estándar 0,12. La frecuencia de la población se asemeja a la curva normal.

A partir de la información entregada por los estadísticos descriptivos, y el gráfico de frecuencias, se procedió a recodificar el Índice de Arraigo Socio-Productivo en:

$$
\begin{aligned}
& 1=\operatorname{MuYBAJO}(0,1 \text { A 0,20) } \\
& 2=\operatorname{BAJO}(0,21 \text { A } 0,40) \\
& 3=\operatorname{Medio}(0,41 \text { A 0,60) } \\
& 4=\operatorname{ALTO}(0,61 \text { A 0,80) } \\
& 5=\operatorname{MuY} \operatorname{Alto}(0,81 \text { A 1). }
\end{aligned}
$$

De los 1329 casos, un 22,7\% tiene un Índice de Arraigo Socio-Productivo de carácter bajo, un 52,8 tiene un Índice de Arraigo Socio-Productivo Medio, mientras los que tienen un alto nivel de Arraigo alcanza el 20\%. Los extremos Muy Bajo y Muy Alto Arraigo Socio-Productivo alcanzan el $2 \%$ respectivamente. 
Si bien la distribución por sexo, en la muestra no es equitativa, las mujeres presentes en ella (231) se ubican en los tramos de Arraigo Socio-Productivo Medio y Bajo, mientras los hombres tienden a agruparse en los tramos de Arraigo Medio y Alto.

Bajo la lógica del Arraigo que aquí se teoriza, aquellas personas que llevan menos tiempo ligados a la actividad tienen una menor identificación, conocen menos a los círculos que las rodean, y por tanto no establecen las mismas relaciones que alguien que lleva más años ligado a una actividad productiva. Si bien, a partir de este estudio no se puede inferir todos estos elementos, efectivamente las personas que llevan más año en la actividad aparecen con índices de Arraigo Alto, sobre todo aquellos que llevan más de 20 años vinculados a la actividad pesquera.

\begin{tabular}{|c|c|c|c|c|c|c|c|c|}
\hline & & \multicolumn{6}{|c|}{ Años ligados a la actividad } & \multirow[t]{2}{*}{ Total } \\
\hline & & 0 a 3 & 4 a 7 & 8 a 11 & 12 a 15 & 16 a 19 & 20 a 51 & \\
\hline \multirow[t]{5}{*}{ Tramos } & Muy Bajo Arraigo & 2 & 0 & 0 & 0 & 0 & 0 & 2 \\
\hline & Bajo Arraigo & 101 & 127 & 64 & 7 & 3 & 0 & 302 \\
\hline & Arraigo Medio & 10 & 77 & 156 & 264 & 108 & 87 & 702 \\
\hline & Alto Arraigo & 0 & 0 & 3 & 30 & 48 & 185 & 266 \\
\hline & Muy Alto Arraigo & 0 & 0 & 0 & 0 & 0 & 3 & 3 \\
\hline \multicolumn{2}{|r|}{ Total } & 113 & 204 & 223 & 301 & 159 & 275 & 1275 \\
\hline
\end{tabular}

Tabla 12. Índice de Arraigo Socio-Productivo por tramos de Años de Experiencia en la Actividad Pesquera

Al mismo tiempo, la Tabla 13 muestra cómo el segmento de la población encuestada que trabaja en las Flotas Artesanal e Industrial son los que presentan un mayor Arraigo Socio-Productivo. Por el contrario, las personas que trabajan en Planta de Reducción, pero sobre todo aquellos que trabajan en Planta de Consumo Humano presentan Índices de Bajo Arraigo. 


\begin{tabular}{|c|c|c|c|c|c|c|}
\hline & & \multicolumn{4}{|c|}{ Segmento muestra SECTOR } & \multirow[b]{2}{*}{ Tota } \\
\hline & & $\begin{array}{c}\text { Flota } \\
\text { Artesanal }\end{array}$ & $\begin{array}{c}\text { Flota } \\
\text { I ndustrial }\end{array}$ & $\begin{array}{l}\text { Planta de } \\
\text { Consumo } \\
\text { Humano }\end{array}$ & $\begin{array}{l}\text { Planta de } \\
\text { Reducción }\end{array}$ & \\
\hline \multirow{5}{*}{$\begin{array}{c}\text { Tramo } \\
2\end{array}$} & Muy Bajo Arraigo & 0 & 0 & 1 & 1 & 2 \\
\hline & Bajo Arraigo & 26 & 19 & 186 & 71 & 302 \\
\hline & Media Arraigo & 122 & 225 & 135 & 220 & 702 \\
\hline & Alto Arraigo & 117 & 100 & 9 & 40 & 266 \\
\hline & Muy Alto Arraigo & 3 & 0 & 0 & 0 & 3 \\
\hline \multicolumn{2}{|r|}{ Total } & 268 & 344 & 331 & 332 & 1275 \\
\hline
\end{tabular}

Tabla 13. Índice de Arraigo Socio-Productivo por Sector donde se emplean en la Actividad Pesquera

Esto puede deberse a la vocación principalmente extractiva que ha tenido la actividad pesquera en su desarrollo histórico, por tanto, la gente que lleva más tiempo en el sector se emplea en la Flotas y la Planta de Reducción. Ligado a lo anterior, puede deberse a que las personas que trabajan en Planta de Consumo Humano tienen una rotación mayor que aquellos que trabajan en la Flota.

\begin{tabular}{|l|l|c|c|c|c|}
\hline \multirow{2}{*}{} & \multicolumn{2}{c|}{ Pertenencia a Sindicatos } & \multirow{2}{*}{} \\
\cline { 3 - 6 } & & Ninguno & Un Sindicato & $\begin{array}{c}\text { Dos } \\
\text { Sindicatos }\end{array}$ & \multirow{2}{*}{ Total } \\
\hline \multirow{3}{*}{$\begin{array}{l}\text { Tramo } \\
\mathbf{2}\end{array}$} & Muy Bajo Arraigo & 2 & 0 & 0 & 2 \\
\cline { 2 - 6 } & Bajo Arraigo & 282 & 19 & 1 & 302 \\
\cline { 2 - 6 } & Arraigo Medio & 377 & 283 & 40 & 700 \\
\cline { 2 - 6 } & Alto Arraigo & 65 & 169 & 32 & 266 \\
\cline { 2 - 6 } & Muy Alto Arraigo & 0 & 2 & 1 & 3 \\
\hline & Total & $\mathbf{7 2 6}$ & $\mathbf{4 7 3}$ & $\mathbf{7 4}$ & $\mathbf{1 2 7 3}$ \\
\hline
\end{tabular}

Tabla 14. Índice de Arraigo Socio-Productivo por Pertenencia a Sindicatos

En términos de participación, el tramo que corresponde a Arraigo Medio tiende a concentrar un número importante de casos en todos los cruces de variables. Ello puede deberse a múltiples factores, pero sin duda lo de más peso pueden ser la desestructuración de las organizaciones de base ocurridas desde la década del 70', como al mismo tiempo, la inserción de la actividad en la Globalización, asociada a una reestructuración del sistema productivo, en el caso de la Pesca a través del Límite Máximo de Captura por Armador, sistema instaurado por la necesidad de manejar el recurso pesquero.

El Bajo Arraigo se asocia a la falta de Participación, pero el Arraigo Medio no, por tanto, es necesario indagar mediante nuevos estudios en la función que cumple la Participación dentro del concepto de Capital Social, ya que aquellos que son miembros de Sindicatos tienen un fuerte Arraigo Socio-Productivo. 
En términos de Participación en otras organizaciones de la sociedad civil, el patrón es bastante similar.

En todas las tablas presentadas el tramo de población que tiene Bajo Arraigo supera el que tiene Alto Arraigo, ello pone de manifiesto que un sector de la población no solo no se "identifica" con la labor productiva que realiza, sino que su relación con la estructura cercana que lo soporta y asiste es débil.

\begin{tabular}{|l|l|c|c|c|c|c|}
\hline \multicolumn{2}{|c|}{} & \multicolumn{4}{|c|}{ Organización de la Sociedad Civil } & Total \\
\hline \multicolumn{2}{|c|}{} & Ninguna & $\begin{array}{c}\text { Una } \\
\text { Organización }\end{array}$ & $\begin{array}{c}\text { Dos } \\
\text { Organizaciones }\end{array}$ & $\begin{array}{c}\text { Tres } \\
\text { Organizaciones }\end{array}$ & \\
\hline Tramo 2 & $\begin{array}{l}\text { Muy Bajo } \\
\text { Arraigo }\end{array}$ & 2 & 0 & 0 & 0 & 2 \\
\hline & Bajo Arraigo & 246 & 52 & 4 & 0 & 302 \\
\hline & $\begin{array}{l}\text { Arraigo } \\
\text { Medio }\end{array}$ & 387 & 292 & 22 & 1 & 702 \\
\hline & Alto Arraigo & 84 & 147 & 30 & 5 & 266 \\
\hline & $\begin{array}{l}\text { Muy Alto } \\
\text { Arraigo }\end{array}$ & 0 & 1 & 1 & 1 & 3 \\
\hline
\end{tabular}

Tabla 15. Índice de Arraigo Socio-Productivo por Pertenencia a Organizaciones de la Sociedad Civil

El que el Arraigo Medio este concentrando a un gran número de casos en la muestra no significa que exista un Capital Social medio, sino que no se ha logrado dinamizar a un sector mayoritario de la población, el cual tiene una relación pasiva con las organizaciones (aunque participe en ellas), con su labor, con sus círculos cercanos y con su propio desarrollo personal.

El Índice muestra que esta población no está ni desarraigada, ni arraigada, por tanto, no cuenta con redes sólidas que abarquen a números importantes de la población, y posiblemente no han desarrollado mayores capacidades comunitarias, como lo muestra la Participación en Organizaciones, donde el Club Deportivo, la Iglesia y, bastante más atrás, la Organización Vecinal, no son capaces de reflejar un peso importante dentro de la idea de un Concepto Amplio de Capital Social.

La pregunta es ¿vale la pena centrar esfuerzos político en fomentar a las organizaciones, principalmente, alejadas de las actividades productivas?, ¿puede generarse un estrategia de desarrollo a partir de la explotación del capital social instalado a través de las actividades productivas?, ¿el identificar los sectores productivos donde la gente este más arraigada permite generar políticas, por ejemplo, por parte del gobierno y las empresas para fomentar el desarrollo de sus trabajadores y del círculo que lo rodea? 


\section{Conclusiones}

Si bien, este trabajo se inspira en un ejercicio exploratorio sobre el concepto de Capital Social, en diferentes tablas de contingencia el Arraigo Medio concentra a la mayoría de la población. Estrictamente hablando, el valor del Índice es más bien Conceptual que Metodológico, ya que representa el como se distribuye la población e incorpora un mayor peso analítico al análisis y desarrollo del Capital Social.

Queda ahora buscar nuevos elementos teóricos y empíricos que fortalezcan el análisis del Arraigo, que es un elemento central a la hora de abordar conceptos como Asociatividad o Desarrollo Endógeno. Para ello hay que realizar nuevas investigaciones que permitan identificar los factores que dinamicen las redes sociales, permitiendo el desarrollo comunitario a partir de la actividad productiva que realizan y aprovechando las estructuras y proto-Instituciones que generan.

El estudio del Arraigo en la literatura Latinoamericana no se ha desarrollado mucho, por lo tanto, se presenta como un terreno fértil para el desarrollo de la sociología, y las ciencias sociales en general. En este trabajo se identificó, por ejemplo, la existencia de brazos cortos y largos en la población estudiada. Esto constituye un punto importante. Hasta ahora, temas de gestión pública, como la participación, el empoderamiento, el desarrollo local, entre otros, han sido vistos desde el Estado, o desde los gobiernos locales, sin embargo, se ha potenciado poco el desarrollo de los Soportes Sociales, verdadero pilar comunitario de ayuda recíproca, que permitiría desencadenar procesos sociales desde una comunidad que se apoya, se organiza y participa.

Analizar que elementos "pesan más" a la hora de identificar y estudiar el Capital Social, permite generar estrategias de fomento e incentivo ligados a actividades productivas. Ello conlleva como punto central, centrar temas como la Participación Ciudadana en el Trabajo, es decir, en las organizaciones y redes que vinculan a las personas con su entorno directo laboral, con su entorno nuclear-familiar e indirecto (comunitario y de brazos largos). De esta forma se le da una perspectiva más territorial al Trabajo, ampliando los campos de acción de las redes sociales.

Parte de la evolución democrática de Chile pasa por incentivar las organizaciones de trabajadores como fuentes de beneficios sociales directos e indirectos para la comunidad, ya que hasta el momento, ni la participación activa en Clubes Deportivos o en Iglesias, ha logrado desencadenar el potencial de la sociedad chilena post-dictadura. 


\section{Bibliografía}

Adler, P.; Kwon, S. (2002). Social Capital: Prospects for a new concept. Academy of Management Review, 27(1): 17-40.

Durston, John (2000), ¿Qué es el capital social comunitario? CEPAL (serie Políticas Sociales, núm. 38). Santiago de Chile:

Granovetter, Mark (2005). The Impact of Social Structure on Economic Outcomes. J ournal of Economic Perspectives. Vol. 19, № 1.Pp. 33-50

Granovetter, Mark (1985). Economic Action and Social Structure. American Journal of Sociology. Vol. 91, № 3. Pp. 481-510.

Granovetter, Mark (1973). La Fuerza de los Vínculos Débiles. American Journal of Sociology. Vol. 78, №6. Pp. 1360-1380.

Kali, R. Social (2003). Embeddedness and Economic Governance: A Small World Approach. Journal of Economic Literature. Classification: L14, 017, D23, P51.

Moody, J y White, D (2003). Structural cohesion and embeddedness: A hierarchical concept of social groups. American Sociological Review. Vol.68, Iss. 1; pg.. 103, 25 pgs.

Parker, Cristián (2001). Capital social y representaciones socioculturales juveniles. Un estudio en jóvenes secundarios chilenos. En Capital social y políticas públicas en Chile. Investigaciones recientes Vol II. Compiladores John Durston y Francisca Miranda. CEPAL, 2001.

Peck, J (2005). Economic Sociologies in Space. Economic Geography. Worcester. Vol.81, Iss. 2; pg. 129, 47 pgs.

PNUD (2000). Desarrollo Humano en Chile.

Polanyi, K (1947). "Nuestra Obsoleta Mentalidad de Mercado". Artículo publicado originalmente en Commentary 13, 1947, 109117. La versión proviene de Polanyi, Art. "Economie primitive, arcaiche e moderne", Giulio Einaudi editore, Turín 1980.

Romero, A., Toledo, X., Romero T, H (2006). Contradicciones socio-territoriales y anclaje (embeddedness) de las actividades turísticas rurales: ejemplos de la vitivinicultura y las actividades forestales en Chile. En Turismo Rural: Patrimonio, Cultura y Legislación. Ed. FACOS - UFSM. Santa María, Brasil.

Serrano, C.; Alarcón, A.; Tassara, G. (2006). “Diseño y Aplicación de Índice Integrado de Capital Social en tres barrios urbanos de la Región de Coquimbo". Programa Más Región. Informe Final. Asesoría para el Desarrollo S.A. 
Uzzi, B y Spiro, J (2005). Collaboration and Creativity: The Small World Problem. American J ournal of Sociology. Vol.111, Iss. 2; pg. 447, 59 pgs.

Uzzi, B (2000). Running Head: Embeddedness and the Economic Performance of Organizations. American Sociological Review. Ms. \#94-289.

Vivo, J.; Hermoso J. y Cano, R. (2005). Factores Latentes del Desarrollo en los Municipios de la Región de Murcia. XII Jornadas de ASEPUMA.

Wellman, B (1995). El análisis estructural: del método y la metáfora a la teoría y la sustancia. Toronto: Universidad de Toronto.

Wellam, B. \& Wortley, S. (1990). Different Strokes from Different Folks: Community Ties and Social Support. American J ournal of Sociology Vol no 96, Nov., 1990: 558-88. 